\title{
Las razones del abandono escolar del bachillerato tecnológico agropecuario
}

\author{
The Reasons for Dropping out in Agricultural \\ High Schools in Mexico
}

Hugo Silva López ${ }^{*}$ y Eduardo Weiss Horz ${ }^{* *}$

\section{RESUMEN}

En este artículo se presentan los resultados de una investigación cualitativa realizada sobre el abandono escolar en cuatro planteles del bachillerato tecnológico agropecuario en México. La investigación se realizó con entrevistas cualitativas a directivos, docentes, tutores, alumnos y jóvenes que abandonaron la escuela. La razón principal ya no es, por la política de becas, económica, sino la reprobación. Ésta se origina por la insuficiencia académica de los estudiantes, el ausentismo en las aulas y la falta de interés en estudiar. Las razones del abandono se encuentran también asociadas a problemas familiares, falta de recursos económicos, desinterés de los padres en el proceso de formación de sus hijos, problemas de salud y embarazos tempranos. En las conclusiones se discute la política de flexibilización de los criterios de acreditación.

Palabras clave: bachillerato tecnológico, alumnos, abandono escolar, México

\section{ABSTRACT}

This article presents the results of a qualitative study carried out about dropping out in four technological/agricultural high schools in Mexico. The research was based on qualitative interviews with principals, teachers, counselors, students, and young people who dropped out of school. The main reason is no longer economic, thanks to the scholarship policy, but failing grades. Failure is associated with academic deficits, absenteeism and lack of interest. Its reasons are also associated with family problems, a lack of economic resources, parental disinterest in their children's education, health issues and teenage pregnancy. Conclusions discuss the policy of flexibilization of accreditation criteria.

Key words: school drop-out, students, agricultural high schools, Mexico

\section{INTRODUCCIÓN}

En el contexto de la obligatoriedad del nivel medio superior en México, el abandono escolar es un problema que ha acaparado la atención de las políticas, de los medios y se ha convertido en foco de acciones de los diferentes actores del sistema educativo. En los últimos diez años se ha realizado un número creciente

\footnotetext{
*Dirección General de Educación Tecnológica Agropecuaria; dgeta@yahoo.com

"Departamento de Investigaciones Educativas del Centro de Investigación y de Estudios Avanzados del Instituto Politécnico Nacional; eweiss@cinvestav.mx

Los autores agradecen la revisión que Enrique Bernal, del DIE-CINVESTAV, hizo al presente trabajo.
} 
de investigaciones sobre el tema; sin embargo, en el bachillerato tecnológico agropecuario el problema ha sido escasamente investigado. El propósito del presente trabajo es presentar los principales resultados de una investigación cualitativa que se realizó en cuatro planteles (pertenecientes a dos estados) con el objeto de describir y comprender las razones del abandono escolar y de uno de sus principales componentes, la reprobación escolar, a partir de entrevistas a alumnos que abandonaron y a alumnos con un gran número de materias reprobadas, de la misma forma que a docentes y directivos. Los resultados de la investigación ponen en entredicho algunos criterios de la política de flexibilización de la acreditación de materias.

Las políticas de inclusión han llevado en la mayor parte de los países de América Latina a establecer la obligatoriedad del nivel medio o secundario. En México, el Senado de la República legisló en 2012 sobre la obligatoriedad de educación media superior, que debe lograrse para el ciclo escolar 2021-2022. El poder ejecutivo se ha propuesto, en el sexenio por concluir, la meta ambiciosa de aumentar la cobertura de la educación media superior de $65.9 \%$ (ciclo 2012-2013) a 86\% en 2018. Con tal fin se han ampliado instalaciones, contratado docentes, establecido contraturnos y servicios escolares nuevos como el Telebachillerato Comunitario y la Preparatoria en Línea. Una de las formas de aumentar la cobertura es "cerrar la llave del abandono", como ha repetido el subsecretario de educación media superior en muchas conferencias. "El abandono escolar en el nivel medio superior alcanzó la tasa de deserción históricamente más alta, de casi 19\%, en el ciclo escolar 1990-1991; 20 años después el índice se ubicó en $15 \%$, es decir, en ese lapso sólo disminuyó cuatro puntos porcentuales". (Tuirán y Hernández, 2015, p. 4). La Subsecretaría de Educación Media Superior (SEMS) se ha propuesto la meta de reducir el abandono escolar en seis ańos por un tercio, de $14 \%$ en 2012 a 9\% en 2018, (CONAEDU, 2015).

Con tal fin, ha convertido el programa SIGUELE del sexenio anterior en el actual Movimiento contra el Abandono Escolar. Ha fortalecido los programas de becas y ha promovido la tutoría, el fomento de habilidades socioemocionales y de seguimiento de los estudiantes en riesgo de abandonar la escuela. Una de las 
medidas centrales es comprometer a los directores de escuelas a elaborar un plan contra el abandono escolar en el que se responsabilicen con determinadas metas a alcanzar (Weiss, 2015). A la vez, la SEMS ha recomendado flexibilizar los procesos administrativos para favorecer la permanencia escolar y la acreditación; en especial asegurarse que las normas y reglamentos de control escolar no limiten las oportunidades de los estudiantes para regularizarse y, en particular, no restringir por cuestiones del ámbito administrativo el número de evaluaciones no semestrales que pueda tomar un alumno en cada periodo o a lo largo de su trayectoria académica, ya sea a través de cursos intersemestrales, exámenes extraordinarios o cualquier otra forma de regularización (CONAEDU, 2015).

El bachillerato tecnológico agropecuario atendía en el ciclo escolar 2013-2014 a una población escolar de 172739 alumnos, que representa $3.6 \%$ de la matrícula total de la educación media superior y $12.3 \%$ de la matrícula total del bachillerato tecnológico. Esta formación actualmente se imparte en 330 planteles. La mayoría de los Centros de Bachillerato Tecnológico Agropecuario (CBTA) fueron creados entre los años 1976 a 1988, con el fin de formar técnicos agrícolas, pecuarios y forestales que fomentaran el desarrollo agropecuario del país. En la estructura curricular de este bachillerato bivalente, la mitad de las horas del plan de estudios se dedicaban, inicialmente, al componente de formación profesional (la mitad de éstas a actividades prácticas), y la otra mitad del plan de estudios al componente propedéutico para permitir continuar estudios de nivel superior a quienes lo desearan. Las sucesivas reformas curriculares al bachillerato tecnológico han aumentado las horas propedéuticas y las comunes entre todos los bachilleratos tecnológicos, mientras que las horas dedicadas a la formación agropecuaria han disminuido. Los planteles fueron fundados en cabeceras municipales de regiones agrícolas, pecuarias y forestales, pero estas cabeceras se encuentran inmersas en un proceso de urbanización creciente. La mayoría de sus estudiantes proviene de familias cuyos ingresos económicos son muy precarios. Sus padres se desempeñan, por ejemplo, como obreros de la construcción, campesinos, empleados estatales; algunas madres de familia que sostienen el hogar son costureras, obreras o empleadas domésticas. 
En los CBTA la deserción escolar pasó de $17.3 \%$ en el ciclo escolar 2006-2007 a 14.7\% en el ciclo escolar 2013-2014. En más de una década la deserción apenas disminuyó 2.6 puntos porcentuales. Parece que los mecanismos de prevención estuvieron ausentes durante muchos años y las acciones para favorecer la permanencia que se llevan a cabo en las escuelas, como veremos, apenas empiezan a influir en el fenómeno del abandono escolar.

Con el propósito de describir el fenómeno de la reprobación y el abandono escolar desde la perspectiva de los diversos actores a nivel de plantel se realizó una investigación cualitativa para tratar de "documentar lo no documentado" (Rockwell, 2011, p. 21). Se buscó indagar en la vida escolar no documentada por los informes burocráticos. Se revisaron los antecedentes de investigación sobre el tema del abandono escolar, mas no se partió de un marco pedagógico, sociológico o psicológico preconcebido, sino se buscó el diálogo entre lo encontrado en las escuelas y referentes bibliográficos pertinentes.

Se entrevistó a los principales actores educativos -directivos, docentes, tutores, alumnos- en cuatro planteles de dos estados. Con apoyo de los tutores, se localizó a jóvenes que habían abandonado la escuela antes de concluir sus estudios en diversos momentos de su recorrido escolar. Al perfilarse en esas entrevistas que la reprobación era una de las causas principales del abandono, se complementó la información con entrevistas grupales a estudiantes irregulares que permanecían en la escuela.

Este artículo se organiza de la siguiente manera: primero se presentan la conceptualización del abandono escolar y los antecedentes de la investigación sobre el tema de las causas, motivos y razones del abandono escolar en el nivel medio superior encontradas por los estudios cuantitativos y cualitativos en México. Luego se describe la metodología utilizada en la investigación cualitativa realizada. A continuación se presentan los hallazgos de esta investigación respecto a las razones del abandono escolar. El tema se trabaja en cuatro apartados, en el primero se mencionan los diversos mecanismos de intervención que los planteles ponen en operación para disminuir el abandono escolar, en el segundo se presentan las razones del abandono escolar expresadas por los jóvenes que abandonaron la escuela, el tercero aborda la pers- 
pectiva de directivos, tutores y docentes y el cuarto profundiza desde la perspectiva de los alumnos irregulares en el tema de la reprobación, uno de los componentes principales del proceso de abandono. En las reflexiones finales se comparan los resultados con otros estudios y se señala un aspecto problemático de la política que busca disminuir el abandono escolar: la flexibilización de la acreditación.

\section{CONCEPTUALIZACIÓN DEL ABANDONO ESCOLAR}

El abandono escolar, un problema educativo importante, es, en primer lugar, tema de las políticas educativas que buscan disminuirlo mediante programas y medidas específicos y en segundo, tema de investigaciones que buscan identificar sus probables causas y valorar el impacto de las políticas para abatirlo. La conceptualización cambiante del tema se puede observar en la denominación, desde el término "deserción escolar" que insinúa una especie de "traición" a la institución escolar. La palabra sigue presente en los índices de la estadística escolar y en ese sentido, se usa también en el presente artículo. El término más neutro y difundido es el de "abandono escolar" que, sin embargo, no considera las interrupciones y reinicios, los "esfuerzos iterativos, recorridos fragmentados" frecuentes entre jóvenes de sectores populares urbanos (Guerra, 2012). Este término sigue ubicando el problema en los alumnos, por eso algunos autores, por ejemplo, Román (2013), oponen el concepto de "expulsión escolar". El término más usado recientemente en el cono sur de América Latina es la "permanencia escolar".

No hay una única teorización fuerte sobre las causas del abandono escolar, aunque se han propuesto abordajes teóricos parciales que en diferentes momentos han tenido mayor o menor difusión. Por ejemplo, la teoría de desafiliación institucional de alumnos que abandonan de Tinto (1992) que ha sido retomada por varios autores, como podremos apreciar en el siguiente apartado sobre los antecedentes de investigación, y el concepto del "desanclaje secular" (Miranda, 2012, p. 71) entre las escuelas, los docentes y los jóvenes que Miranda sintetiza con la frase: "escuelas del siglo XVIII, los maestros del siglo XIX y los estudiantes del 
siglo XXI", concepto que inspira parte de nuestras conclusiones. Se puede observar cierto movimiento desde teorías que situaban las causas del abandono en factores macroesctructurales y en la situación socioeconómica de las familias hacia enfoques que lo ubican en el ambiente escolar y la interacción educativa (Tuirán y Hernández, 2015).

Los trabajos empíricos, en su mayoría de carácter cuantitativo, no suelen hablar de causas sino de factores asociados o predictores probables del abandono escolar (Rumberger y Lim 2008; SEP, 2012). La causalidad en sentido estricto sólo puede establecerse mediante experimentos, y en los análisis estadísticos cuasi-experimentales sólo se constatan asociaciones más o menos fuertes y constantes; la atribución de causalidad a estas asociaciones depende de la teorización que la soporte.

Los factores de los estudios cuantitativos suelen basarse en encuestas que arrojan dos tipos de información, "motivos para desertar que manifiestan quienes responden el cuestionario; por otra parte, se obtienen datos importantes ... propios del ambiente en el que viven las personas encuestadas" (SEP, 2012, p. 5). Las entrevistas en estudios cualitativos suelen buscar motivos (los para qué de las acciones) o razones (los porqué de las acciones). Cabe mencionar que los dos términos son reversibles, lo que es hoy un motivo para mi actuar mañana se convierte en razón de mi acción.

La mayoría de los estudios coinciden en que el abandono escolar es un proceso multifactorial, complejo, dinámico y acumulativo (Abril, Román, Cubillas y Moreno, 2008; Artismuño, 2009; Maya, 2012; Román, 2013; Tuirán y Hernández, 2015; Székely, 2015), las "variables consideradas no tienen un efecto aislado sobre la probabilidad de desertar, sino que por el contrario, el efecto de estos factores es interactivo y multiplicativo" (Miranda 2012, p. 75). Estudios realizados en la línea de investigación Jóvenes y escuela en el Departamento de Investigaciones Educativas (Guerra, 2012; Weiss, 2012) muestran que los jóvenes viven ciertas situaciones, realizan prácticas que se entrelazan entre sí en un proceso y generan situaciones críticas (hitos) que pueden derivar en el abandono de la escuela. 


\section{ANTECEDENTES DE LA INVESTIGACIÓN}

Las investigaciones sobre el tema del abandono escolar suelen comprender un gran número de factores y razones, que pueden agruparse en diferentes categorías. El metaestudio de 208 investigaciones en Estados Unidos realizado por Rumberger y Lim (2008) categoriza entre factores individuales (desempeño académico, comportamiento, actitudes y antecedentes) e institucionales (características de las familias, de las escuelas y de las comunidades). Muchos estudios distinguen entre factores exógenos y factores endógenos (como los llama Román, 2013) o factores extraescolares e intraescolares (SEP, 2012). También se distinguen factores a diferentes niveles de organización. Román (2013) distingue estructurales, político-institucionales y culturales; Székely (2015), en una revisión sistemática de diversos informes nacionales e internacionales, clasifica por factores individuales y familiares, factores de la comunidad y factores macro. El estudio más amplio realizado en México, la Encuesta Nacional de Deserción en la Educación Media Superior (SEP, 2012) realizada en 2011 con una muestra representativa de 2000 "desertores" categoriza: a) el contexto del individuo y familiar; b) el ámbito educativo: relación con la escuela y con la educación, interacción con profesores, expectativas educativas, y c) el entorno del entrevistado y las condiciones socioeconómicas. Nosotros presentamos la revisión de los antecedentes de investigación bajo las siguientes dimensiones: económica o social, académica o educativa y familiar o personal. Revisamos y sistematizamos 29 estudios realizados en México de corte cuantitativo y cinco de enfoque cualitativo que abordan el problema del abandono escolar en la educación media superior.

Razones económicas. La mayoría de los estudios sostienen que las razones económicas son una de las principales causas del abandono escolar. Algunos estudios las reportan de manera genérica: Navarro (2001) señala que 35\% de los jóvenes encuestados desertaron por causas económicas; Abril et al. (2008) que 37\% de los encuestados consideró los factores económicos como causa de deserción. La Subsecretaría de Educación Media Superior (SEMS, 2010) con datos obtenidos en la encuesta de contexto aplicada a 
directores de planteles en la prueba ENLACE (Evaluación Nacional de los Logros Académicos en los Centros Escolares) señala que $43 \%$ de los directores de las escuelas del nivel medio superior reportaron como causa de abandono los problemas económicos; la SEMS (2015) indica que 38\% de los encuestados abandonó la escuela porque "faltaba dinero en el hogar"; Navarro (2011) calcula que del universo de encuestados, 33\% mujeres y $42 \%$ hombres, abandonaron la escuela por motivos económicos.

Otros estudios especifican las razones, principalmente, los gastos que implican la escuela y la necesidad de trabajar y aportar de esta manera dinero a la casa: el Instituto Nacional de Estadística y Geografía (INEGI, 2009) a través de la Encuesta Nacional de Ocupación y Empleo (ENOE) registra que 52\% de los encuestados señaló la "insuficiencia de dinero para pagar la escuela" y la "necesidad de aportar dinero al hogar". La SEP (2012) a través de la Encuesta Nacional de Deserción en la Educación Media Superior (ENDEMS) reporta que 36.4\% de los encuestados mencionó "la falta de dinero en el hogar para útiles, pasajes o inscripción", y 7.2\% "consideraba trabajar más importante que estudiar"; Ruiz, García y Pérez (2014) encuentran que 17.7\% de los encuestados respondió que "faltaba dinero para la inscripción", 14\% expresó "no tener dinero para comprar libros y $11.4 \%$ "tener que trabajar”. El Instituto Mexicano de la Juventud (IMJ, 2005) con los datos obtenidos de la Encuesta Nacional de Juventud, indica que $42 \%$ de los que dejaron de estudiar lo hizo porque "tenía que trabajar"; Székely (2015) señala que los factores macro, hacen referencia a mejores oportunidades laborales, las cuales incrementan el costo de oportunidad de permanecer en la escuela.

Razones escolares o académicas. Las razones escolares o académicas son otra causa importante del abandono escolar presentada en los estudios revisados. La razón principal suele ser porque "no quisieron o no les gustó estudiar": 37\% (Navarro, 2001); 29\% (IMJ, 2005); 11\% (INEGI, 2009).

Es de notarse que esta razón podría agruparse también como personal; sin embargo, su relación con lo escolar y académico aparece cuando se desglosa: el IMJ (2010) encuentra que de $42.6 \%$ de los encuestados que seńalaron que el abandono escolar se debió a razones escolares, $15.9 \%$ mencionó que le aburría la 
escuela, $13.1 \%$ no quiso estudiar y $9.6 \%$ respondió que reprobaron materias. La SEP (2012), con datos de la ENDEMS, indica que la segunda razón de abandono escolar fue que "le disgustaba estudiar"; le siguen: con 7.8\% "le dieron de baja por reprobar materias", con $6 \%$ por problemas para entender a los maestros y con $7.1 \%$ un "turno distinto al que quería". INEGI (2009) reporta que $2.5 \%$ abandonó por "reprobación, suspensión o expulsión"; Ruiz et al. (2014) refieren que los factores escolares son la principal causa del abandono escolar, $31.4 \%$ respondió "reprobé materias", $22.9 \%$ por "falta de interés en estudiar", $11.4 \%$ por "malas calificaciones”. La SEMS (2010) identificó, en una encuesta, que los directores reportaron que $24 \%$ de los estudiantes abandonan por la falta de interés en la escuela y $19 \%$ por bajo rendimiento escolar. Como podemos apreciar, además del disgusto por el estudio aparecen problemas para entender a los maestros, el aburrimiento, malas calificaciones y reprobaciones (que a su vez pueden ser causa de la falta de interés por los estudios).

En la Encuesta Nacional de Deserción en la Educación Media Superior (SEMS, 2012) las razones académicas del abandono son las siguientes: "no asiste regularmente a la escuela", "no le parecen interesantes los estudios", "los estudios piensa que no le van a brindar nada", "ha reprobado repetidamente o en varias materias"; Székely (2015) establece que el desempeño académico, repetir año y el ausentismo escolar son factores que inciden en la decisión de abandonar la escuela.

Estudios más bien cualitativos, como el de Vidales (2009), profundizan en las razones académicas a través de la identificación de factores relacionados con el ambiente y la gestión escolar que inciden en el rendimiento de los estudiantes, como por ejemplo "la situación de los docentes y su poca profesionalización"; "alta carga de alumnos por grupo", pero también "deficiencias en la formación de los estudiantes en temas como matemáticas, habilidades cuantitativas y verbales, y conocimiento del español".

Gómez y Miranda (2010) señalan que la deserción es multifactorial, los jóvenes manifestaron que hay una falta de conexión con el ambiente escolar, se percibe que la escuela es aburrida, se sienten desmotivados o no pueden con los retos académicos. " $\mathrm{La}$ escuela aparece ante los jóvenes como un campo de ausencias y 
rigideces institucionales: currículos inflexibles, horarios rígidos, maestros cerrados y burocracias panópticas, sin contar con la ofensiva de los sistemas de evaluación, la reprobación y el fracaso escolar" (Miranda, 2012, p. 80).

Razones personales y/o familiares. Las razones que agrupamos aquí tienen que ver, por un lado, con la falta de apoyo de las familias para que los jóvenes estudien (que en parte va asociada a condiciones económicas) y por otro, con embarazos o matrimonios de los jóvenes; en menor medida también con problemas como la drogadicción y el alcoholismo.

El IMJ (2005) reporta que $10.6 \%$ no se inscribió en la escuela "por cuidar a la familia” y $12.1 \%$ "porque los padres ya no quisieron”. Ruiz et al. (2014) concluyen que la tercera causa del abandono escolar fueron los factores familiares: 5.7\% mencionó que "mi papá no me apoyó para seguir estudiando", 5.7\% "mi pareja no me deja seguir estudiando", $5.7 \%$ expresó que "la relación con mi papá o mamá es inestable”.

Otra razón importante es el embarazo, el matrimonio o la unión con una pareja. Navarro (2001) calculó que el 5.8\% de los jóvenes que abandonan la escuela lo hacen "porque se casó o se unió"; INEGI (2009) indica que 12\% de los encuestados que abandonaron la escuela lo hicieron por embarazo, matrimonio y unión libre; IMJ (2010) registra que 17\% de los jóvenes abandonan por embarazo. La ENDEMS (SEP, 2012) reporta que la razón "se embarazó, embarazó a alguien o tuvo un hijo" sólo era seńalada por $4.7 \%$ de los encuestados como la principal, pero por $17.5 \%$ como una de las tres principales y al desagregar la encuesta por género se observa que $23.8 \%$ de las mujeres señalaron que "se embarazó o tuvo un hijo" y $22.9 \%$ que "se casó" como las dos razones que siguen en importancia a la falta de dinero en el hogar.

Székely (2015) considera que la principal causa de abandono deriva de factores individuales y familiares; también agrupa condiciones socioeconómicas y culturales como la educación de los padres, la estructura familiar y ser parte de una minoría, a la vez que señala el uso de drogas, consumo de alcohol y el embarazo adolescente que afectan la probabilidad de seguir estudiando.

Entre los estudios con enfoque cualitativo revisados, Guerra (2012) señala, basado en relatos de vida, tres tipos de estudiantes 
que se desafiliaron de la institución escolar: los que optaron por el trabajo o el matrimonio; los que tuvieron conflictos con la escuela y dejaron truncos sus estudios; los que desarrollaron una relación de rechazo a la escuela y al trabajo y optaron por la vida en grupos juveniles. Estrada (2014), basado en entrevistas biográficas e historias de vida de jóvenes desertores de tres escuelas de nivel medio superior, señala que la reprobación es la principal causa del abandono, pero sólo cuando desencadena un alto grado de desafiliación a la escuela.

\section{METOdOLOGÍA}

Para llevar a cabo la investigación cualitativa, se seleccionaron cuatro planteles atendiendo los siguientes criterios:

Por razones de costos de la investigación se seleccionaron dos estados cercanos a la ciudad de México, uno con baja deserción con $12 \%$ en promedio (Hidalgo) y otro con alta deserción con $22 \%$ en promedio (Morelos).

Con base en un análisis de la estadística oficial que proporcionó la Dirección General de Educación Tecnológica Agropecuaria (DGETA) sobre el abandono escolar e índices de reprobación y eficiencia terminal ordenada por estado y plantel, se seleccionaron dos escuelas de cada una de las entidades federativas mencionadas, una con un porcentaje alto de deserción y otra con bajos índices. Asimismo, se consideró que una de estas escuelas se ubicara en un contexto rural y otra que tuviera características más urbanas (cuadro 1).

Las escuelas del bachillerato tecnológico agropecuario se orientan al ámbito rural; desde su origen fueron ubicadas en cabeceras municipales que, por su número de habitantes, se clasifican como urbanas. Actualmente en tres de ellas hay un creciente proceso de transición rural-urbana (El Tephé, Apán y Huitzilac). Tetela del Volcán conserva más sus características rurales. Como se puede ver en el cuadro 1, en Huitzilac hay notablemente más deserción y reprobación y en Tephé, menos. 
CUADRO 1. Muestra intencional de escuelas seleccionadas

\begin{tabular}{|l|c|c|c|c|}
\hline \multicolumn{1}{|c|}{ Estados } & \multicolumn{2}{|c|}{ Hidalgo } & \multicolumn{2}{c|}{ Morelos } \\
\hline \multicolumn{1}{|c|}{ Criterios } & Escuela 1 & Escuela 2 & Escuela 3 & Escuela 4 \\
\hline Ubicación & El Tephé & $\begin{array}{c}\text { Ex Hacienda San Juan } \\
\text { Ixtilmaco }\end{array}$ & Huitzilac & $\begin{array}{c}\text { Tetela del } \\
\text { Volcán }\end{array}$ \\
\hline Índice de marginación & Medio & Bajo & Bajo & Medio \\
\hline Matrícula 2012-2013 & 1709 alumnos & 1060 alumnos & 453 alumnos & 536 alumnos \\
\hline $\begin{array}{l}\text { Índice de deserción } \\
\text { 2012-2013 }\end{array}$ & $8 \%$ & $18 \%$ & $27 \%$ & $8 \%$ \\
\hline $\begin{array}{l}\text { Índice de reprobación } \\
\text { 2012-2013 }\end{array}$ & $39 \%$ & $26 \%$ & $48 \%$ & $35 \%$ \\
\hline Pertenece al SNB* & Nivel III & Nivel II & Nivel III & Nivel III \\
\hline
\end{tabular}

*SNB. Sistema Nacional de Bachillerato es un esquema diseñado para la evaluación de planteles, creado por la Secretaría de Educación Pública. Los estándares e indicadores de evaluación están clasificados por niveles ordenados del IV que el nivel más bajo hasta el I que es el nivel más alto.

Fuente: elaboración propia.

En el trabajo de campo se visitaron los planteles seleccionados. En cada visita se efectuaron entrevistas en profundidad (Taylor y Bodgan, 1986) a una muestra intencional de los diferentes informantes. La parte más difícil fue localizar a los jóvenes que abandonaron la escuela; ellos fueron ubicados con el apoyo de la dirección de las escuelas a través de los responsables de vinculación y entrevistados en sus casas; se entrevistó a 14 jóvenes. El análisis preliminar mostró que una de las causas principales del abandono era la reprobación; por ello se decidió complementar la información con ocho entrevistas grupales a estudiantes irregulares que permanecían en la escuela. Además, se realizaron cuatro entrevistas a directores de plantel, cuatro a subdirectores académicos, ocho a jefes de programas de tutorías y orientación educativa, diez a tutores y 25 entrevistas a docentes de asignaturas de matemáticas, lectura, expresión oral y escrita, física, química, inglés y módulos profesionales. Se elaboraron los registros y las transcripciones, al mismo tiempo que se realizaban análisis parciales de la información obtenida (Rockwell, 2011). Posteriormente, se agrupó la información por temas utilizando el software Atlas-ti y se elaboraron tablas comparativas. 


\section{RESULTADOS}

\section{Las acciones de las escuelas para disminuir el abandono escolar}

En las escuelas visitadas funcionan diversos mecanismos generales de intervención para disminuir el abandono escolar, como la participación en el programa de becas, la organización del Movimiento contra el abandono escolar, dentro del que se desarrollan tutorías, orientación educativa, reunión con padres de familia, talleres para padres de familia y alumnos en riesgo (El Tephé), escuela para padres (Tetela del Volcán), talleres de habilidad matemática y habilidad lectora (Huitzilac y Tetela del Volcán). Además, participan en el programa Construye $\mathrm{T}$, promovido por el Programa de las Naciones Unidas para el Desarrollo (PNUD) y la Subsecretaría de Educación Media Superior: en cada plantel se realizan actividades para mejorar el ambiente escolar y desarrollar las competencias socioemocionales.

Las medidas que tomaron las cuatro escuelas para prevenir el abandono escolar son similares, si bien en Tetela del Volcán hay una actividad más. El análisis tampoco encontró diferencias notables entre los planteles en términos de las razones para el abandono y la reprobación. Por ello los resultados del abandono escolar se presentan en los apartados que siguen de manera generalizada para los cuatro planteles.

La diferencia más notable entre los planteles -y probablemente causa de las diferencias en las tasas de abandono- se encuentra en el prestigio y la política de admisión de las escuelas. Al bachillerato tecnológico agropecuario en Huitzilac, la escuela con el índice más alto de reprobación (47\%) y de deserción (28\%) -véase cuadro 1-asisten muchos estudiantes que no fueron aceptados en otros bachilleratos de la localidad; en cambio, el bachillerato agropecuario de El Tephé, con una tasa de reprobación de $39 \%$ pero con una tasa de deserción de sólo 8\%, puede escoger a sus estudiantes en un proceso de selección. Sin embargo, esto es apenas una hipótesis, el diseño de la presente investigación es insuficiente para probarla. 
La investigación encontró en los planteles indicios de que habían seguido las recomendaciones de sus autoridades educativas y habían flexibilizado la normatividad escolar ofreciendo más opciones a los estudiantes para poder permanecer en la escuela. En un caso, la flexibilidad no se aplicó a un alumno que por enfermedad no asistió a presentar los exámenes, fue reprobado y en consecuencia tuvo que abandonar la escuela.

En un estado, la coordinación estatal autorizó un acuerdo de coordinadores de no reprobar más de $10 \%$ de la matrícula por asignatura y módulo. Una medida cuestionable que discutiremos en las conclusiones.

\section{Razones del abandono escolar: jóvenes que abandonaron la escuela}

En el cuadro 2 se muestran las razones por las que los jóvenes abandonaron la escuela antes de concluir sus estudios y se puede apreciar la combinación de las razones, así como la frecuencia con la que expresaron determinada razón.

De los 14 entrevistados, siete abandonaron durante o después del primer semestre, dos estaban en segundo, cuatro en $4^{\circ}$ y uno incluso en $6^{\circ}$ semestre. Él había reprobado cuatro asignaturas; una asignatura del tercer semestre y otra del quinto semestre, y estaba reprobado en dos asignaturas del sexto semestre.

La tabla muestra, en primer lugar, que casi siempre hay múltiples razones para el abandono escolar. La reprobación aparece como la razón más frecuente en 12 de los 14 casos presentados.

La reprobación se relacionaba en todos con el ausentismo en el aula: "no entraba a clases" o "por faltas". Reconocían también "no entregar trabajos", "se me olvidaba entregar tareas", o "no me gustaba". Cuatro de los 12 mencionaron: "no entendía y no ponía atención”, "no entiendo, va muy rápido”. Dos jóvenes más que no mencionaron la reprobación como causa, señalaron la falta de interés en el estudio. (Profundizaremos más adelante en el tema de la reprobación). 
LAS RAZONES DEL ABANDONO ESCOLAR DEL BACHILLERATO TECNOLÓGICO AGROPECUARIO

CUADRO 2. Razones de los jóvenes que abandonaron la escuela

\begin{tabular}{|c|c|c|c|c|c|c|c|}
\hline Nombre & Semestre & Reprobación & $\begin{array}{c}\text { Falta de } \\
\text { interés en el } \\
\text { estudio }\end{array}$ & $\begin{array}{l}\text { Problemas } \\
\text { familiares }\end{array}$ & $\begin{array}{l}\text { Falta de } \\
\text { recursos } \\
\text { económicos }\end{array}$ & $\begin{array}{l}\text { Desatención de } \\
\text { los padres en } \\
\text { el proceso de } \\
\text { formación }\end{array}$ & $\begin{array}{l}\text { Problemas } \\
\text { de saludy } \\
\text { embarazo }\end{array}$ \\
\hline Felipe & $6^{\circ}$ & $\bullet$ & & $\bullet$ & $\bullet$ & - & \\
\hline Nereyda & $1^{\circ}$ & $\bullet$ & & $\bullet$ & $\bullet$ & $\bullet$ & \\
\hline Gonzalo & $4^{\circ}$ & $\bullet$ & & $\bullet$ & $\bullet$ & & \\
\hline Amparo & $2^{\circ}$ & $\bullet$ & & $\bullet$ & $\bullet$ & & \\
\hline Elia & $4^{\circ}$ & $\bullet$ & & - & $\bullet$ & $\bullet$ & \\
\hline Emmanuel & $1^{\circ}$ & $\bullet$ & & - & & • & \\
\hline Yessica & $1^{\circ}$ & $\bullet$ & & $\bullet$ & & & \\
\hline Daniel & $1^{\circ}$ & $\bullet$ & & - & & & \\
\hline Ángel & $2^{\circ}$ & $\bullet$ & $\bullet$ & & & & $\bullet$ \\
\hline David & $1^{\circ}$ & $\bullet$ & & & & & $\bullet$ \\
\hline Alisson & $4^{\circ}$ & $\bullet$ & & & & & $\bullet$ \\
\hline Marian & $4^{\circ}$ & $\bullet$ & & & & & - \\
\hline Alfredo & $1^{\circ}$ & & - & & & & \\
\hline Aldo & $1^{\circ}$ & & • & & & & \\
\hline
\end{tabular}

Fuente: elaboración propia con información obtenida de las entrevistas individuales.

La reprobación se encuentra asociada a otras razones. En ocho de los 14 casos se presentaron problemas familiares que se combinan en cinco de los ocho con la falta de recursos económicos y en tres con la desatención de los padres en el proceso de formación. Este hallazgo es significativo ya que permite inferir que los problemas familiares causan problemas económicos a la vez que no sólo implican aspectos económicos. Con relación a los problemas familiares comentaron en las entrevistas: "Me salí de mi casa, porque tuve problemas con mi padrastro", "vivo con mi mamá, tuve problemas con mi papá", "tengo problemas familiares", "mi mamá se fue a los Estados Unidos", "a mi papá no lo conozco", "mi papá se separó". Veamos algunos casos de problemas familiares:

Gonzalo, de 18 años, vivía solo, sus papás se separaron, su papá se fue para los Estados Unidos. Su mamá vive en otro lado, de vez en cuando se visitan. Había cursado el cuarto semestre y 
llevaba cuatro asignaturas reprobadas, asistía a la escuela, pero no entraba a clases. Trabajaba los fines de semana en un rancho agrícola.

Emmanuel, un chico de 15 años, tuvo dificultades con su padrastro y tomó la decisión de irse de su casa. Su ausencia frecuente en las clases lo llevó a reprobar materias, más allá de las permitidas. Para continuar sus estudios, tuvo que abandonar la escuela; expresó que regresará al CBTA en el próximo semestre. Se dedicaba a trabajar en una imprenta.

Amparo, de 17, que aspiraba terminar la preparatoria, pero sus problemas de ausentismo y reprobación, así como problemas familiares (la separación de sus padres) afectaron su permanencia en la escuela. Se dedicaba a trabajar en una lonchería los fines de semana, mientras esperaba reingresar al sistema abierto.

La falta de recursos económicos afectó a un tercio de los que abandonaron la escuela, los jóvenes expresaron "no vengo (a la escuela) porque no me alcanza" (Felipe), o "es que no venía a la escuela, es que también a mi mamá no le alcanza para mi pasaje" (Nereyda). Ella tiene 16 años, dejó de asistir a la escuela y a clases y reprobó por atender a su hermano enfermo por encargo de su mamá. Tenía la intención de volver al CBTA en el siguiente semestre; por lo pronto, se quedó en su casa a ayudar en las labores domésticas.

Estos problemas económicos se presentan a pesar de que entre 80 y $95 \%$ de los estudiantes están becados por programas federales, estatales y del propio plantel. Por otro lado es de destacarse que, a diferencia de la gran mayoría de estudios-que reseñamos en el apartado de antecedentes- y que señalan las razones económicas como una de las causas principales, en éste sólo un tercio de los que abandonaron señalan problemas económicos. Es de suponerse que la política de becas ha contribuido a mejorar la situación.

La falta de interés de los padres de familia en el proceso de formación de sus hijos contribuyó al abandono en cuatro casos, esta razón estuvo asociada a problemas familiares y falta de recursos económicos. Los jóvenes mencionaron que "citaron a mis papás, pero no han venido, no han dado respuesta” (Elia), "cuando citaban a mi mamá, casi no venía" (Emmanuel), "cuando citaban a mi mamá, no venía a la escuela” (Felipe). 
En el cuadro 2, se puede apreciar que hay un grupo de cuatro estudiantes que tuvo problemas de salud y de embarazo, que se combinaron con los de reprobación, mas no con problemas familiares ni económicos. Los tres casos con problemas de salud eran: depresión, varicela y diabetes. Daniel comentó: "Dos semanas antes de las evaluaciones me dio varicela, estuve dos semanas en mi casa. Cuando regresé ya habían pasado los exámenes, ya no tuve oportunidad de presentarlos, tuve que salir de la escuela". En este caso se observa una falta de flexibilidad en la aplicación de la reglamentación escolar. Alisson de 18 años, tenía la intención de permanecer en la escuela, pero una depresión le impidió asistir con regularidad a sus clases y solicitó su baja. Se encontraba en su casa apoyando a su mamá en el negocio familiar mientras se atendía de su enfermedad para poder regresar a la escuela. Su madre expresó la expectativa de que su hija regresara al CBTA.

El embarazo fue causa de abandono en un caso, una exalumna comentó: "Cometí una locura, me junté con mi novio, me embaracé y me detectaron una enfermedad y mi pareja ya no me deja estudiar, ahora soy ama de casa, estoy viendo a ver si estudio el sistema abierto, pero no sé".

\section{La perspectiva de directivos, jefes de programa, docentes y tutores}

En las entrevistas realizadas, los directivos, jefes de programa, docentes y tutores coinciden en señalar la reprobación, la falta de interés en los estudios, la falta de recursos económicos, los problemas familiares, los problemas de salud o embarazos y la desatención de los padres de familia en el proceso de formación de sus hijos como razones del abandono escolar.

A continuación profundizaremos, en palabras de los entrevistados, en los significados de estas razones.

Falta de recursos económicos: Para los entrevistados, la pobreza relacionada con los bajos ingresos económicos son claros determinantes del abandono escolar, en algunos casos muy ligados a la necesidad de trabajar por parte del estudiante. La mayoría de los estudiantes proviene de familias cuyos ingresos económicos son muy precarios. Tal como se mencionó antes, sus 
padres se desempeñan como obreros de la construcción, campesinos, empleados estatales; algunas madres de familia que sostienen el hogar son costureras, obreras o empleadas domésticas.

En la expresión de los docentes y tutores: "Muchachitos que en vacaciones trabajan, te dicen: 'Yo me gano la vida barbechando la tierra.' Y faltan una semana, faltan tres días y así se la llevan; van dos, tres semanas bien y luego empiezan a fallar, son irregulares". "Este joven trabaja sábados y domingos, se pone a ayudarle a su mamá porque pagan renta".

Problemas familiares: Los directivos, docentes y tutores expresaron que muchos jóvenes estudiantes viven solos, no cuentan con alguien que se preocupe por ayudarlos o les facilite los recursos y orientación para realizar sus estudios. Los principales problemas que afectan la vida de los alumnos son los divorcios y la desintegración familiar. Un tutor dice: "Estamos manejando casi un $60 \%$ de esos muchachos con problemas familiares muy importantes, que no les permiten a ellos concentrarse en su actividad principal, que es la escuela".

Desinterés de los padres en el proceso de formación de sus hijos: La participación de los padres en la educación de sus hijos juega un papel muy importante. Sin embargo, muchas veces se desentienden de sus hijos y "transfieren" su obligación a la escuela de manera general o a los docentes de manera particular. Una docente comentó: "Si los alumnos no cooperan y los papás no te ayudan, qué vamos hacer. Te cansas, no hay eco".

Problemas de salud y embarazo: Los problemas de salud pueden representar una crisis en la vida de los jóvenes y de sus familias. Una ausencia temporal o prolongada de la escuela por enfermedad, frecuentemente induce a decidir abandonarla. El embarazo precoz se presenta como una razón para que las alumnas abandonen la escuela. Estas situaciones se incrementan a pesar de la información disponible, que quizás no funciona por el difícil acceso a los anticonceptivos y a su uso correcto. Un docente señala su alta recurrencia, en el semestre anterior "el $12 \%$ de los estudiantes desertores fue por embarazo".

Falta de interés en el estudio: El abandono escolar responde también a intereses y decisiones originados por la desmotivación, como la falta de interés en estudiar o por priorizar otros ámbitos 
o espacios en su vida. Los directivos, docentes y tutores comentaron que el desinterés por el estudio se presenta porque: "Hay mucha apatía", "no quieren estudiar", "no les gusta". "Por problemas de no agrado, no interés, el muchacho no está en clases; dice: 'no me gusta entrar a clases, no se me hace divertido, no se me hace entretenido'; entonces creo que por ahí hay mucha reprobación producto de la inasistencia; quiere decir: 'no asisto, entonces, no participo, no trabajo, no creo, no me involucro, no me integro.' Entonce, no hay evidencia, no hay productos con qué evaluarlo".

\section{Razones de la reprobación según alumnos irregulares y adultos a cargo del proceso educativo}

Dada la importancia del fenómeno de la reprobación se realizaron entrevistas grupales a alumnos irregulares que estaban rezagados en sus asignaturas y en riesgo de abandonar la escuela. En todos los planteles visitados había estudiantes rezagados o irregulares, algunos arrastraban asignaturas de semestres anteriores y llevaban también asignaturas reprobadas del semestre que cursaban (como vemos en el cuadro 3). El Reglamento General de Control Escolar para el Bachillerato Tecnológico. Ciclo Escolar 2014-2015. Planteles Oficiales de la DGECyTM, DGETA, DEGETI y CECYTE, establece que debe haber tres reportes parciales que sustentan la evaluación final. Al momento de la visita, ya había pasado la evaluación del segundo parcial y las asignaturas que hasta ese momento llevaban reprobadas los ponían en riesgo de abandonar la escuela, si en el tercer parcial no mejoraban sus promedios. En los planteles visitados, en promedio, tres de cada diez estudiantes tenían asignaturas reprobadas, la mayoría eran del primer semestre. Los alumnos irregulares comentaron que han reprobado por ausentismo ("no entraban a clases", "por faltas"), "por no entregar tareas" y por no acreditar exámenes; a veces también por mal comportamiento dentro del aula ("el profe me sacó de la clase”) o por enfermedades. La principal razón del ausentismo y no entregar trabajos y tareas es la falta de interés en el estudio ("le dije a mi novia que no quería estudiar, y se lo dijo a mi mamá, pero mi mamá me mandaba a la escuela”), pero también algunos 
mencionan que "no entendía". En el cuadro 3 se aprecia que las asignaturas más reprobadas por los alumnos eran matemáticas, inglés, tecnología de la información y la comunicación, lectura, expresión oral y escrita, lógica, ciencia, tecnología, sociedad y valores, módulo agropecuario.

Las razones de la reprobación que aparecen en la tabla son:

- Ausentismo en todos los casos, cuya principal expresión fue "no entrar a clases", en parte no asistían a la escuela, en parte iban a la escuela, pero no entraban a clases; esas ausencias se presentaban en las asignaturas que más reprobaban los muchachos. Al acumular determinadas inasistencias, el sistema de control escolar, de manera automática (que señala que el alumno debe cubrir $80 \%$ de asistencia para tener derecho a evaluación), los reprueba.

- Falta de interés en casi todos los casos; se manifiesta en no entregar trabajos ni tareas que forman parte del proceso de evaluación de las asignaturas. No entender la explicación de contenidos fue una expresión que manifestaron cuatro alumnos al referirse a los contenidos de matemáticas, inglés y lectura, expresión oral y escrita.

- Actitud en el aula en un caso; por el mal comportamiento dentro del salón de clases, el docente lo sacaba y no le permitía entrar; en consecuencia, acumulaban faltas y no entregaba trabajos ni tareas.

- No acreditar exámenes: dos estudiantes comentan no haber pasado los exámenes parciales de las asignaturas.

- Por enfermedad. En un caso no asistía a clases, acumulaba faltas y no entregaba tareas. 
LAS RAZONES DEL ABANDONO ESCOLAR DEL BACHILLERATO TECNOLÓGICO AGROPECUARIO

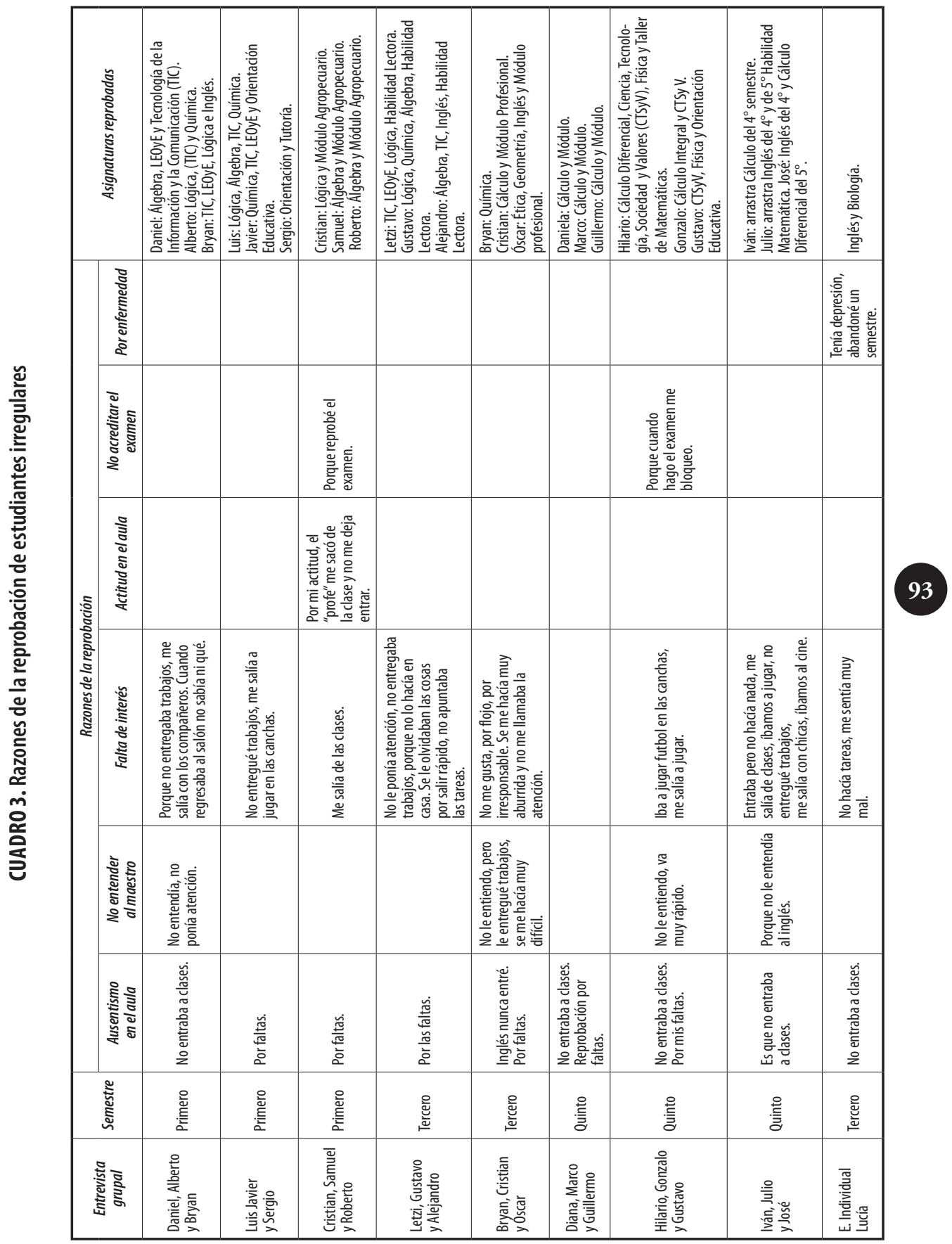

Hugo Silva López y Eduardo Weiss Horz 
Por su parte, los directivos, docentes y tutores consideran que la reprobación se debe a la insuficiencia académica de los estudiantes y al ausentismo en las aulas, pero también incluyen razones ajenas a los alumnos, como el ausentismo de docentes, la planeación didáctica deficiente, que no toma en cuenta a los alumnos, el sistema de control escolar y los parámetros de evaluación del aprendizaje. Ahondaremos a continuación en estos temas expresados por los entrevistados:

a) La insuficiencia académica de los estudiantes: La reprobación es producto de las insuficiencias académicas que tienen los alumnos desde la secundaria. En los planteles visitados las asignaturas que más reprobaron los estudiantes fueron: química, inglés, matemáticas, física, biología, lectura, expresión oral y escrita y lógica. Un docente dice: "Son estudiantes que en la secundaria los acreditan sin hacer nada; entonces, esto ya es un problema que viene desde la educación básica y que lamentablemente en donde quieren que se les alinee es en la educación media superior". En un examen diagnóstico realizado por la DGETA (2017) en el ciclo escolar 2016-2017, estudiantes de nuevo ingreso obtuvieron en matemáticas 3.5 de calificación y en lectura 3.6.

b) Ausentismo en las aulas: Una docente comentó: "En la escuela hay mucho ausentismo por cualquier motivo, que les duele la cabeza, que si hay lluvia ya no vienen.” Y luego están los estudiantes que no entran a clases. El ausentismo refleja la falta de interés de los estudiantes. El desinterés se debe a múltiples razones. También se origina en la presión que ejercen los padres de familia para que sus hijos estudien. Hay casos de alumnos cuyos padres los obligan a asistir a la escuela. Un alumno comenta: "ellos quieren que estudie, pero yo no quiero, yo no quiero seguir estudiando, pero mis papás me obligan”.

c) Ausentismo de docentes: Se presenta por comisiones académicas, comisiones sindicales, días económicos o prestaciones laborales. Las horas académicas no se recuperan. Los estudiantes pierden el ritmo del aprendizaje y se desmotivan. Los estudiantes "de cierta manera pierden el interés de la escuela por nosotros mismos". 
d) La planeación didáctica no toma en cuenta a los alumnos: Algunos docentes reconocen que la planeación didáctica y las prácticas pedagógicas que realizan algunos profesores están asociadas a la expulsión de los estudiantes. Un docente comentó: "Creo que los docentes no hemos tenido la capacidad de que la planeación didáctica sea acorde a las necesidades de los muchachos. Pocas veces consulto a los muchachos para desarrollar mi planeación, es decir, qué quieren aprender, cómo quieren aprender, a qué ritmo lo quieren aprender. No tomamos en cuenta ni el contexto ni los estilos de aprendizaje de los muchachos. Creo que ésa es la problemática que está provocando la reprobación".

e) El control escolar: El sistema tecnológico en general cuenta con un mecanismo institucionalizado de control escolar denominado Sistema de Servicios Escolares de la Educación Media Superior (SISEEMS), que se ha convertido en un problema porque, al registrar el incumplimiento de $80 \%$ de asistencia a clases, genera la reprobación y en determinados casos hasta el abandono escolar.

f) Parámetros de evaluación del aprendizaje: De manera general, en los planteles los docentes valoran el avance de los alumnos en forma paralela al proceso de aprendizaje, sin usar técnicas especializadas de evaluación. Calculando rangos entre lo que dijeron los profesores entrevistados, la evaluación se basa $30 \%$ en exámenes, de 10 a $50 \%$ en el portafolio o la presentación de trabajos y tareas, y en 10 o $20 \%$ en la participación. $\mathrm{Al}$ requerir a los alumnos presencia y cumplimiento cotidiano de tareas, en muchos casos los estudiantes no alcanzan a cubrir estas exigencias, que son parte de la evaluación de las asignaturas.

\section{REFLEXIONES FINALES}

La decisión de abandonar la escuela es un proceso asociado a una diversidad de problemáticas que experimentan los jóvenes en el transcurso de su vida cotidiana y trayectoria escolar. El presente estudio cualitativo confirma para el bachillerato tecnológico agropecuario lo que han señalado casi todos los estudios 
sobre el tema (SEP, 2012; Román, 2013; Tuirán y Hernández, 2015; Székely, 2015): que el abandono escolar no se produce por una causa aislada, sino por múltiples razones, las más de las veces entrelazadas entre sí. En las escuelas estudiadas el abandono escolar aparece vinculado a la reprobación, al ausentismo, a problemas familiares, a problemas económicos y a problemas de salud o de embarazo.

A diferencia de la mayoría de estudios (Navarro, 2001; Abril et al., 2008; SEMS, 2010; INEGI, 2009; SEP, 2012; SEMS, 2015; Székely, 2015), que señalan la situación económica y la necesidad o el interés por trabajar como una de las causas principales del abandono escolar, en esta investigación sólo fueron mencionados por un tercio de los estudiantes que abandonaron. Es probable que esto se deba a que entre 80 y $95 \%$ de los estudiantes cuentan con beca; también hay que tomar en cuenta que los estudiantes que tienen que trabajar o gustan de trabajar ya abandonaron la escuela antes de iniciar el bachillerato; asimismo, hay pocos empleos disponibles actualmente.

En cambio, apareció un tema poco enfatizado en los estudios acerca del abandono escolar: los problemas familiares como los divorcios y la desintegración familiar. Muchas veces se oye hablar a los docentes de problemas familiares de los estudiantes como causa de las dificultades escolares, pero los investigadores educativos desestiman su importancia y suelen considerarlo como una forma de los profesores de echar la culpa a factores extraescolares. También incidió otro tema poco mencionado en los estudios, los problemas de salud, que impiden a los estudiantes cumplir con la asistencia y con las tareas que exigen las asignaturas, por ello algunos reprueban más materias de las permitidas por la normatividad de control escolar.

En el presente estudio la reprobación destaca como la principal razón del abandono escolar. A su vez, la reprobación se relaciona fuertemente con el ausentismo y el ausentismo con la falta de interés por estudiar, que fue señalada tanto por los profesores como por los propios estudiantes. Los profesores señalaron, además, las insuficiencias académicas que los estudiantes portan desde el nivel previo. Lo confirman los resultados de la prueba PLANEA (Plan Nacional para la Evaluación de los Aprendizajes, 
SEP, 2016) que la SEP aplica en el nivel medio superior a los estudiantes que cursan el sexto semestre, y en la que la mitad o más de los alumnos de tercero de secundaria tienen logros insuficientes de los aprendizajes clave del currículum, $46 \%$ en lenguaje y comunicación y $65 \%$ en matemáticas. Los estudiantes mismos no hablaron de eso, si bien algunos mencionaron "no entendí al profesor". Podemos suponer que las insuficiencias académicas dificultan la comprensión y que la falta de comprensión influye en la falta de interés. El ausentismo en las aulas, es decir estudiantes que asisten a la escuela, pero no se meten a las aulas, va en aumento. En algunos casos los padres de familia obligan a los hijos a asistir a la escuela, aunque éstos no tengan interés, sea para que no permanezcan en casa sin hacer nada, o porque la beca es complemento del sustento familiar.

Resulta significativo que 20 de 25 docentes entrevistados reconocen no sólo las deficiencias de los estudiantes, sino también las ausencias de algunos colegas y que "no hemos tenido la capacidad de que la planeación en el aula sea acorde a las necesidades de los muchachos". Sin embargo, sorprende que los maestros no cuestionen el plan y los programas de estudio que son demasiado extensos y de carácter propedéutico para el estudio a nivel superior, mientras ofrecen pocas horas de práctica.

En el bachillerato tecnológico hay un sistema computacional que reprueba a los alumnos si no cuentan con $80 \%$ de asistencia a las clases y con dos asignaturas reprobadas, en automático, quedan fuera del plantel. Las autoridades educativas han flexibilizado la normatividad otorgando más opciones para regularizarse y los profesores ya no son estrictos con la asistencia, pero el sistema computacional sigue funcionando y vimos un caso de ausentismo por enfermedad que no debía haber llevado a la reprobación. Para disminuir cifras de abandono algunos planteles y autoridades tomaron la decisión de no reprobar más de $10 \%$ de los estudiantes por asignatura, una traducción pragmática, desde la lógica escolar burocrática, de la obligación de la Subsecretaría de fijar y lograr determinadas metas de permanencia de los estudiantes. A nuestro ver, esta medida, como pudimos también observar en otro estudio (Saccone y Weiss, 2017), induce a la simulación de la enseñanza y del aprendizaje: los estudiantes saben que con 
un mínimo esfuerzo de "echarle ganas", pueden obtener una calificación aprobatoria y los docentes exigen cada vez menos. El bachillerato, más que en un lugar de aprendizaje y de formación, se convierte en lugar de contención social de los jóvenes, como ha señalado también Miranda (2012). Superar este problema exige una reforma curricular profunda.

\section{REFERENCIAS BIBLIOGRÁFICAS}

Abril, E., Román, R., Cubillas, M.J. y Moreno, I. (2008). ¿Deserción o autoexclusión? Un análisis de las causas de abandono escolar en estudiantes de educación media superior en Sonora, México. Revista Electrónica de Investigación Educativa, 10(1). Recuperado de http://redie.uabc.mx/ vol10no1/contenido-abril.html

Artismuño, A. (2009). El abandono de los estudios del nivel medio en Uruguay: Un problema complejo y persistente. Revista Iberoamericana sobre calidad, eficacia y cambio en educación, 7(4),180-197. Recuperado de http://www.rinace.net/reice/numeros/arts/vol7num4/art9.pdf

Consejo Nacional de Autoridades Educativas (CONAEDU), (2015). LII Reunión del Consejo Nacional de Autoridades Educativas-Capítulo EMS. México: SEP. Recuperado de http://www.sems.gob.mx/work/models/sems/Resource/12125/52_CONAEDU_EMS.pd.

Dirección General de Educación Tencológica Agropecuaria (2017) Evaluación del ingreso al bachillerato. Ciclo escolar 20016-2017. México.

Estrada, M. (2014). Afiliación juvenil y desafiliación institucional. El entramado complejo de la deserción en la educación media, Revista Mexicana de Investigación Educativa, 19(61), 431-453.

Gómez, L. y Miranda, F. (2010). Modelo integral para la atención y acompańamiento de adolescentes y jóvenes de la educación media superior. Informe Final. México: FLACSO.

Guerra, M.I. (2012) Recorridos escolares. Jóvenes de sectores populares y escuela: encuentros y desencuentros a lo largo de la vida, pp. 243-266. En Eduardo Weiss (Coord.) Jóvenes y Bachillerato, México. ANUIES. 
Instituto Mexicano de la Juventud (IMJ), (2005). Encuesta Nacional de Juventud 2005. Resultados preliminares, México. Recuperado de http://sic.conaculta.gob.mx/centrodoc_documentos/292.pdf

Instituto Mexicano de la Juventud (IMJ), (2010). Encuesta Nacional de la Juventud. Recuperado de http://www. imjuventud.gob.mx/imgs/uploads/Encuesta_Nacional_de_ Juventud_2010_-_Resultados_Generales_18nov11.pdf

Instituto Nacional de Estadística y Geografía (INEGI), (2009). Encuesta Nacional de Ocupación y Empleo (ENOE), México: INEGI.

Maya, C.(2012). El abandono escolar: Una perspectiva sociocultural para su interpretación. México, UPN. 320 pp.

Miranda, F. (2012). Los jóvenes contra la escuela. Un desafío para pensar las voces y tiempos para América Latina, $R e$ vista Latinoamericana de Educación Comparada, México, FLACSO. México, 71(3), 71-84. Recuperado de http:// www.saece.org.ar/relec/revistas/3/art6.pdf

Navarro, A. (2011). Educación, pobreza y desigualdad en el bachillerato mexicano, México: Centro de Estudios Sociales y de Opinión Pública, Documento de trabajo, pp.1-127.

Navarro, N. (2001). Marginación escolar en los jóvenes. Aproximación a las causas de abandono. Notas. Revista de información y análisis, INEGI, 15,43-50. Recuperado de http:// www.inegi.gob.mx/inegi/contenidos/espanol/prensa/ contenidos/articulos/sociodemograficas/marginacion.pdf

Rockwell, E. (2011). La experiencia etnográfica. Historia y cultura en los procesos educativos. Buenos Aires, Argentina: Editorial Paidós.

Román, M. (2013). Factores asociados al abandono y la deserción escolar en América Latina: Una mirada en conjunto. Revista Iberoamericana sobre Calidad, Eficacia y Cambio en Educación, 11(12), 39-59. Recuperado de http://www. rinace.net/reice/numeros/arts/vol11 num12/art2.pdf

Ruiz, R. García, J.L. y Pérez, M.A. (2014). Causas y consecuencias de la deserción escolar en el bachillerato: Caso Universidad Autónoma de Sinaloa. Revista Ra Ximhai, 
10 (15), 51-74. Recuperado de http://www.redalyc.org/ articulo.oa?id=46132134004

Rumberger, R.W. y Lim, A.S. (2008). Why students drop out of school: a review of 25 years of research. California Dropout Research Project, Report 15. Recuperado de http://www.slocounty.ca.gov/Assets/CSN/PDF/Flyer++Why+students+drop+out.pdf

Saccone, M. y Weiss, E. (2017). La acreditación de asignaturas en la vida cotidiana escolar. Un estudio etnográfico en el contexto de la obligatoriedad de la educación media superior. Propuesta Educativa, 47, 119-128.

Secretaría de Educación Pública (SEP).(2012). Reporte de la Encuesta Nacional de Deserción en la Educación Media Superior (ENDEMS). México: SEP.

Secretaría de Educación Pública (2016). Prueba PLANEA. Recuperado de http://www.sems.gob.mx/PLANEA2016.

Secretaría de Educación Pública (SEP). (2014). Reglamento General de Control Escolar para el Bachillerato Tecnológico. Ciclo Escolar 2014-2015. Planteles oficiales de la DGECyTM, DGETA, DGETI y CECYTE. México: SEP-SEMS.

Subsecretaría de Educación Media Superior (SEMS).(2010). El abandono escolar en la educación media superior. Presentación.

Subsecretaría de Educación Media Superior (SEMS). (2015). Análisis del Movimiento Contra el Abandono Escolar en la Educación Media Superior. México: SEMS-Instituto Nacional de Salud Pública.

Székely, M. (2015). Estudio sobre los principales resultados y recomendaciones de la investigación y evaluación educativa en el eje de prevención y atención a la deserción escolar en educación media superior. Informe Final. México.

Taylor, S.J. y Bogdan, S. (1986). Introducción a los métodos cualitativos de investigación. La búsqueda de significados. Buenos Aires, Argentina: Editorial Paidós.

Tinto, V. (1992). El abandono de los estudios superiores. Una nueva perspectiva de las causas del abandono y su tratamiento. México: ANUIES. 
Tuirán, R. y Hernández, D. (2015). El abandono escolar en el bachillerato. Revista Este País. Tendencias y opiniones, 20, 5-10. Recuperado de http://www.estepais.com/articulo. php?id=197\&t=el-abandono-escolaren-el-bachillerato

Vidales, S. (2009). El fracaso escolar en la educación media superior de una universidad mexicana. Revista Iberoamericana sobre Calidad, Eficacia y Cambio en Educación, 7(4), 320341. Recuperado de http://www.rinace.net/reice/numeros/arts/vol7num4/art16.pdf

Weiss, E. (2012). (Coord.). Jóvenes y Bachillerato. México: Asociación Nacional de Universidades e Instituciones de Educación Superior.

Weiss, E. (2015). El abandono escolar en la educación media superior: dimensiones, causas y políticas para abatirlo. En: R. Ramírez (Coord.). Los desafios de la educación media superior. México: Senado de la República. 